
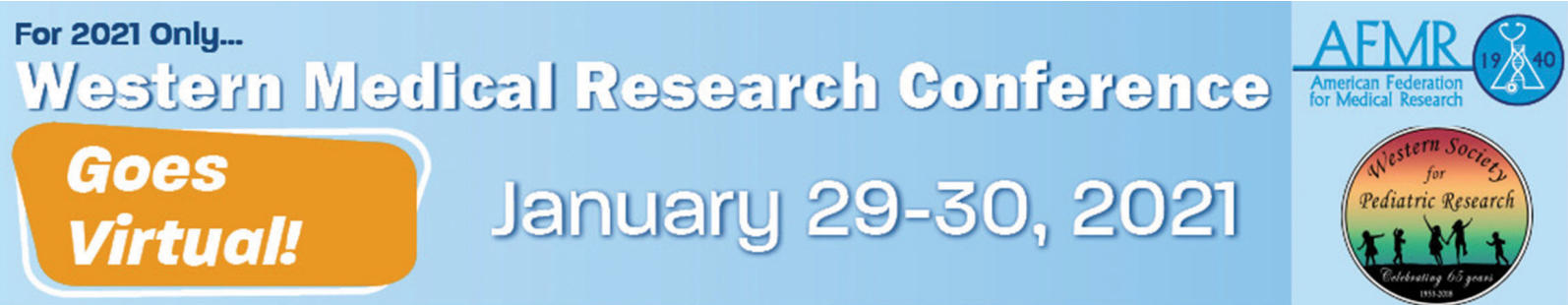

\title{
2021 Western Medical Research Conference
}

\section{AWARD WINNERS}

\begin{tabular}{|c|c|}
\hline Shinya Abe, Kobe University Graduate School of Medicine & WSPR Mead Johnson Merit Award \\
\hline Geoanna Bautista, University of California Los Angeles & AFMR Henry Christian Award \\
\hline Dana Button, Oregon Health \& Science University & WAFMR Subspecialty Award \\
\hline Shanlee Davis, University of Colorado & WSPR Abbott Nutrition Young Investigator Research Award \\
\hline $\begin{array}{l}\text { Tristan Dear, University of Colorado Denver School of } \\
\text { Medicine }\end{array}$ & WSPR Outstanding Resident Research Award \\
\hline Evgenia Dobrinskikh, University of Colorado & WSPR Mead Johnson Merit Award \\
\hline Theodore Gross, Valley Children's Hospital & WAFMR Subspecialty Award \\
\hline Adina Hazan, Cedar's-Sinai Medical Center & WAFMR Subspecialty Award \\
\hline Sarah Kee, University of Washington & WAFMR Subspecialty Award \\
\hline $\begin{array}{l}\text { Lauren Lee, University of California Los Angeles David } \\
\text { Geffen School of Medicine }\end{array}$ & $\begin{array}{l}\text { WSPR Abbott Nutrition Lowell Glasgow Student Research } \\
\text { Award }\end{array}$ \\
\hline $\begin{array}{l}\text { Rebecca Levy, Stanford University School of Medicine, } \\
\text { Stanford University School of Medicine }\end{array}$ & WSPR Mead Johnson Merit Award \\
\hline $\begin{array}{l}\text { Olivia Man, University of California Los Angeles David } \\
\text { Geffen School of Medicine }\end{array}$ & WAFMR Subspecialty Award \\
\hline Pranay Maniar, New Jersey Institute of Technology & WAFMR Subspecialty Award \\
\hline Monica Penon Portmann, UCSF, UCSF & WSPR Mead Johnson Merit Award \\
\hline $\begin{array}{l}\text { Hannah Peterson, Loma Linda University School of } \\
\text { Medicine }\end{array}$ & WAFMR Subspecialty Award \\
\hline Jonathan Repine, University of Colorado & WAFMR Subspecialty Award \\
\hline Caitlin Ritz, University of Colorado & WAFMR Subspecialty Award \\
\hline Amitoj Singh, University of California San Francisco & WAFMR Subspecialty Award \\
\hline $\begin{array}{l}\text { Boris Stepanyuk, University of Colorado Denver School of } \\
\text { Medicine }\end{array}$ & WSPR Mead Johnson Merit Award \\
\hline Katie Strobel, University of California-Los Angeles & WSPR Abbott Nutrition David W, Smith Award \\
\hline $\begin{array}{l}\text { Tania Torbati, Western University of Health Sciences } \\
\text { College of Osteopathic Medicine of the Pacific, } \\
\text { Cedars-Sinai Medical Center }\end{array}$ & WAFMR Subspecialty Award \\
\hline Alicia White, University of Colorado & WSPR Mead Johnson Merit Award \\
\hline
\end{tabular}

